\title{
Experimental study of the LED lamp
}

\author{
Marek Markowicz ${ }^{1}$,Emil Smyk ${ }^{2^{*}}$, and Robert Smusz ${ }^{3}$ \\ ${ }^{1}$ Faculty of Mechanical Engineering and Aeronautics, Rzeszow University of Technology, \\ al. Powstańców Warszawy 12, 35-959 Rzeszów, Poland \\ ${ }^{2}$ Faculty of Mechanical Engineering, UTP University of Science and Technology, \\ al. Prof. S. Kaliskiego 7, 85-796 Bydgoszcz, Poland \\ ${ }^{3}$ Faculty of Mechanical Engineering and Aeronautics, Rzeszow University of Technology, \\ al. Powstańców Warszawy 12, 35-959 Rzeszów, Poland
}

\begin{abstract}
LEDs are widely used light sources. Their main advantages are low power consumption, mechanical strength, high luminous efficacy, and a lifetime exceeding 50000 hours. However, compared to other light sources, they are more sensitive to high temperatures. Heat is generated by converting electrical energy into radiant energy. The share of heat in this transformation describes the efficiency, but in the case of light sources, efficiency is not one of the basic parameters because only a part of the radiation produced is visible light, which interests the recipients. However, the temperature has a big influence on the properties and lifetime of LEDs. This article presents the results of temperature measurements of the LED lamp.
\end{abstract}

\section{Introduction}

In recent years there has been a dynamic development of the technology of producing light sources. This made LEDs become light sources with properties acceptable for typical applications. The increase in luminous efficacy and the increased luminous flux from a single LED chip make semiconductor radiation emitters effectively replace classic incandescent light sources and become strong competition for fluorescent sources.

LEDs are semiconductor structures. They consist of an n-type semiconductor layer, a p-type semiconductor layer, an active area called the p-n junction, and a pair of metal contacts: the positive electrode and the negative electrode. The basis of its operation is electroluminescence. A characteristic feature of material $p$ is having an excess of holes in the valence band, while material $\mathrm{n}$ has an excess of electrons in this band. When the diode is polarized in the direction of conduction, electrons, and holes penetrate into the active layer with a lower energy level. In the p-n junction, the energized electrons recombine with the holes and get rid of the excess energy, which is radiated as a quantum of light (photon emission). The value of the energy of the emitted photon is approximately equal to the gap between the energy states, characteristic of the analyzed semiconductor material.

LEDs have many advantages compared to other light sources. The main advantages are low power consumption, compact size, mechanical strength, ease to change the color and

${ }^{*}$ Corresponding author: emil.smyk@utp.edu.pl 
intensity of lighting, high luminous efficacy, and lifetime, often exceeding 50000 hours [1]. In the case of LEDs, the lifetime is defined more rigorously than in the case of other light sources. Namely, it is defined as the time in which the luminous flux drops to $70 \%$ of its initial value, while in the case of classic light sources, the term average lifetime is used, in which the value of the luminous flux will decrease by $50 \%$. The main reason for this approach is the fact that LED modules (e.g. PCBs with diodes and current stabilizers installed) in most cases are treated as non-replaceable and therefore should ensure the proper functioning of the lamp throughout its lifetime.

The dynamic properties of LEDs much better than other commonly used light sources make them more susceptible to fluctuating current and voltage, so powering LED lamps is more complicated, especially when powered from an AC mains. This is because a single LED will only conduct current in one direction at a voltage of a few volts. The current-voltage characteristic of the LED diode requires forcing the current flowing through the diode, and not the voltage at its terminals. In the case of a voltage supply, small changes in voltage would cause large changes in the flowing current, and thus in the luminous flux produced. In addition, LEDs are characterized by low inertia, which is a disadvantageous property when used in lighting technology. When the current flowing through the diode is interrupted, its lighting is almost instantly lost. In the case of a $50 \mathrm{~Hz}$ mains supply, this will result in a distinct flickering, which we will practically not observe with traditional light bulbs.

The LED is a converter of electrical energy into radiant energy. This efficiency can be significant. The most modern LEDs have an energy efficiency of 50\%. This means that more than $50 \%$ of the supplied energy is converted into radiation, and less than $50 \%$ is lost in the form of heat, which must be given up to the environment. However, in the case of light sources, efficiency is not one of the basic parameters given by manufacturers. Users are not interested in the power of radiation, but in the light impression. This describes the luminous flux. It can be said that the luminous flux describes the total amount of visible light produced. The unit of the luminous flux is the lumen. An ordinary LED diode can produce a luminous flux of e.g. 2, 20, or 2001m, while even the best IRED diode will always produce a luminous flux of $01 \mathrm{~m}$ because it emits lightly invisible to the eye (IRED diode will produce radiation of a certain power, but the luminous flux will be zero). To distinguish from classical efficiency, in the case of visible light, the concept of luminous efficacy is used, expressed in lumens per watt $(1 \mathrm{~m} / \mathrm{W})$, meaning the resultant ratio of the obtained luminous flux to the electric power supplied to the diode. Leading manufacturers of LEDs report that their laboratories produce LEDs with luminous efficacy exceeding $300 \mathrm{~lm} / \mathrm{W}$ (at a color temperature of $5150 \mathrm{~K}$ ), which gives an energy efficiency of $50 \%$. This significantly exceeds the efficiency of incandescent light, halogen, and even HID (Hyper Intensive Discharge) bulbs, making LEDs one of the most efficient light sources [2].

In common opinion, LEDs are energy-saving, high-efficiency, durable, reliable, and resistant to mechanical shock light sources. But obtaining such good results in real operation is possible under several conditions, the most important of which is adequate heat dissipation. The photometric properties and lifetime of LEDs largely depend on the temperature of the LED junction. The increase in temperature of the LED junction causes a decrease in the luminous flux and excessive degradation of the diode chip structure. Research [3] shows that about $70 \%$ of LED damage is caused by excessively high junction temperature, and changes in luminous flux are a function of luminophore degradation under the influence of increased temperature. Furthermore, LEDs generate more heat as they age, e. g. [4].

LEDs have a higher percentage of visible light compared to incandescent, fluorescent, and metal halide lamps. Unfortunately, they are also characterized by having a high value of the non-radiant heat energy component [5] that can raise the junction temperature to a value that causes damage to the LED. That is why effective heat dissipation is so important [6]. 
There are two cooling technologies applied to LEDs, namely: passive cooling and active cooling. In the case of passive cooling, no additional power consumption is required and its operation is based on the use of natural convection. They are many ways to use passive cooling, such as the use of an optimally designed heat sink [7], the use of two-phase closed thermosyphons, the use of vapor chambers [8], the use of phase-change material modules [9], mounting a fin-type heat sink, liquid cooling [10] and many others. Unfortunately, as the power of LEDs increases, the size of the elements used for passive cooling increases, which causes e.g. higher production costs. In addition, when passive techniques are used to manage larger power levels, phase-change systems such as thermosyphons or heat pipes are required [6]. Therefore, in recent times, an increase in the popularity of active cooling techniques and their development can be observed. Active cooling techniques are based on the use of forced convection via the liquid or gaseous medium. Liquid cooling, such as single-phase [11], multi-phase flows [12,13], nanofluids [14], direct spray [15], and others, provides significant cooling possibilities but requires the use of additional components, e.g. reservoirs, pumps, spray systems, etc., which makes the whole the process becomes expensive and complicated. Thus, in recent years we have observed a great development of active air-cooling methods, such as cooling with piezo fans or cooling with synthetic jets. Piezo fans consist of flexible shim attached to a piezoelectric patch. The piezoelectric patch generates a flapping motion at the shim tip during oscillation, and the resulting air currents are used to cool the heated surface [16]. This type of cooling is mainly used for cooling small regions of electronics [17]. Whereas the technology of the synthetic jet in its basic configuration consists of a synthetic jet actuator - the cavity with the orifice in the one wall, and with one wall replaced by the diaphragm. The oscillating diaphragm at the opposite end of the cavity creates an unsteady airflow into and out of the cavity. The diaphragm creates pairs of vortex rings near the exit from the orifice, which increases heat transfer. Synthetic jets are particularly useful in the LED cooling systems and their technology is constantly being developed [18-21].

The aim of the article is to study the characteristic temperatures close to the LEDs, to test the active power, and to measure the average heat transfer coefficient from the LED lamp surface.

\section{Materials and methods}

The subject of the research was LED lamp model HB4-III-NW-DT-120 (Fig. 1) submitted from Luxon for research purposes. The LED lamp has a nominal power of $153 \mathrm{~W}$, the nominal luminous flux is $24500 \mathrm{~lm}$ and the color temperature is $4000 \mathrm{~K}$. The supply voltage was $230 \mathrm{~V}$.

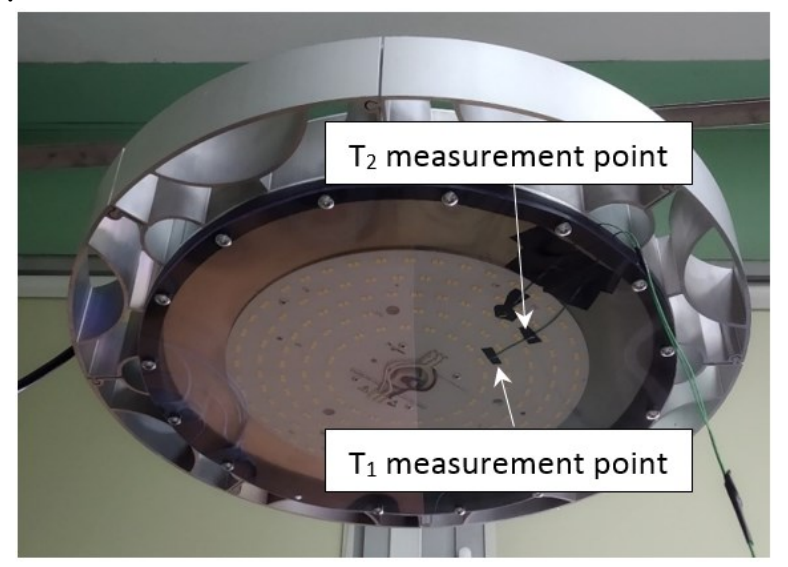

Fig. 1. The tested LED lamp with a nominal power of $153 \mathrm{~W}$, with installed temperature sensors 
Temperature measurement was made using two K-type thermocouples attached to a PCB close to SMD LEDs. The thermocouples are connected to a Testo 400 measuring instrument. The air temperature was measured with an additional Pt100 resistance sensor also connected to Testo 400. This meter enables the recording of measurement data with a sampling frequency of $2 \mathrm{~Hz}$. The heat transfer coefficient was determined using a heat flux sensor with a built-in thermocouple type Omega HFS-3. The LED lamp was connected to the GWINSTEK GPM-8213 power analyzer, which allows the active, reactive and apparent power to be measured by the power receiver. Figure 2 shows a photo of the test stand.

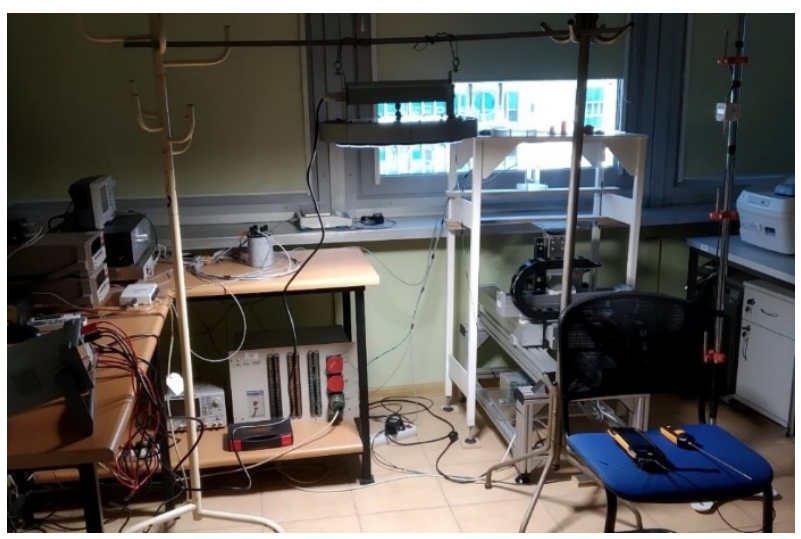

Fig. 2. Photo of the test stand

\section{Results and discussion}

Figure 3 shows the time course of temperatures after switching on the LED lamp. In Figure $3 \mathrm{a}$ the row data was presents and in Figure $3 \mathrm{~b}$ the data after processing and their approximation was showed. As can be seen, the course of the temperature curves $T_{1}$ and $T_{2}$ were logarithmic, and the temperature curves $\mathrm{T}_{\text {air }}$ was linear (mean value $23.36 \pm 0,16^{\circ} \mathrm{C}$ ).

a)

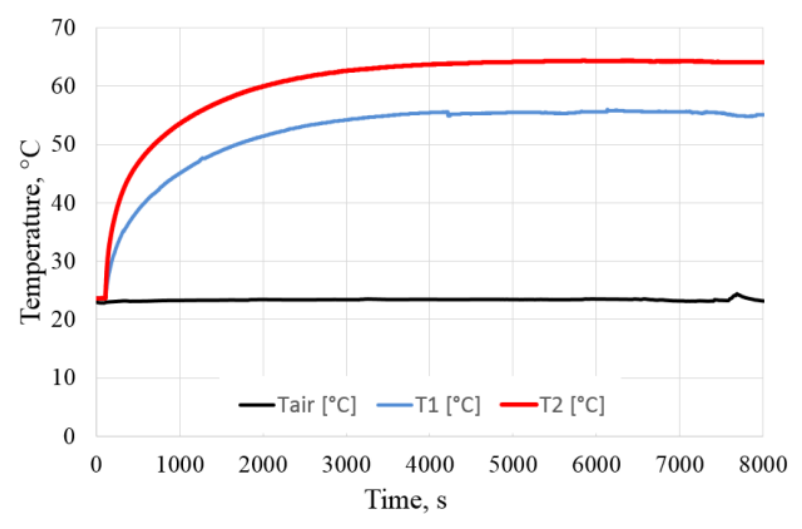

b) 


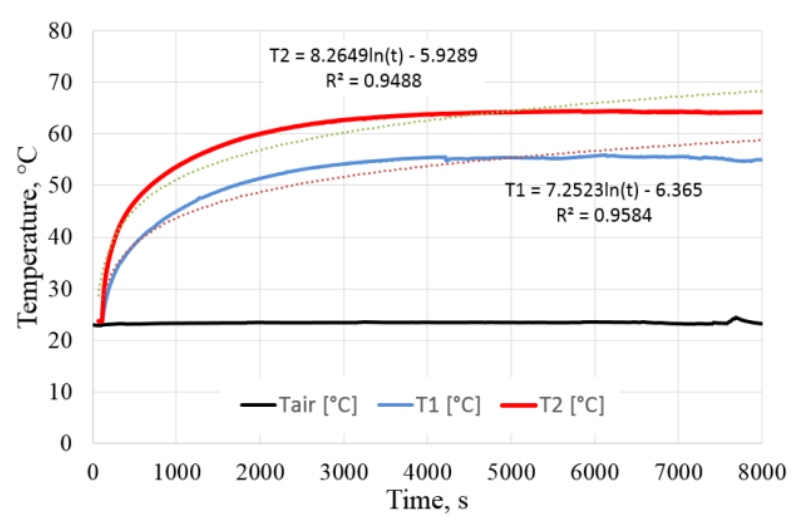

Fig. 3. Time course of temperatures after switching on the LED lamp for row data(a) and approximation of results after after data processing (b)

In the tested LED lamp, the time for the temperature to settle at $90 \%$ of the maximum value is about 40-50 minutes and is approximately equal to reaching the steady-state. Using Newton's equation the thermal power given off by the housing of the LED lamp was estimated as $Q=107,08 \mathrm{~W}$. The parameters of LED lamp supply and thermal parameters were presented in Table 1.

Table 1. Measured values

\begin{tabular}{|l|c|c|}
\hline Properties & Conditions & Units \\
\hline Active power & 147 & $\mathrm{~W}$ \\
\hline Thermal power & 107,08 & $\mathrm{~W}$ \\
\hline Power factor & 0,996 & - \\
\hline Voltage & 229,5 & $\mathrm{~V}$ \\
\hline Voltage frequency & 50 & $\mathrm{~Hz}$ \\
\hline Maximum temperature & 64,3 & ${ }^{\circ} \mathrm{C}$ \\
\hline Air temperature & 23,4 & ${ }^{\circ} \mathrm{C}$ \\
\hline Housing temperature & 36,1 & ${ }^{\circ} \mathrm{C}$ \\
\hline Heat transfer coefficient & 16,63 & $\mathrm{~W} / \mathrm{m}^{2} \mathrm{~K}$ \\
\hline Surface area & 0,507 & $\mathrm{~m}^{2}$ \\
\hline
\end{tabular}

It must be noted that the same LED lamp was tested in [18]. Gil [18] calculated the thermal power of the lamp for two cases: free forced convection. In the case of free convention, Gil [18] used the heat sink other than that supplied by the lamp manufacturer. The comparison of our research results and results presented by Gil [18] was presented in fig. 4. 


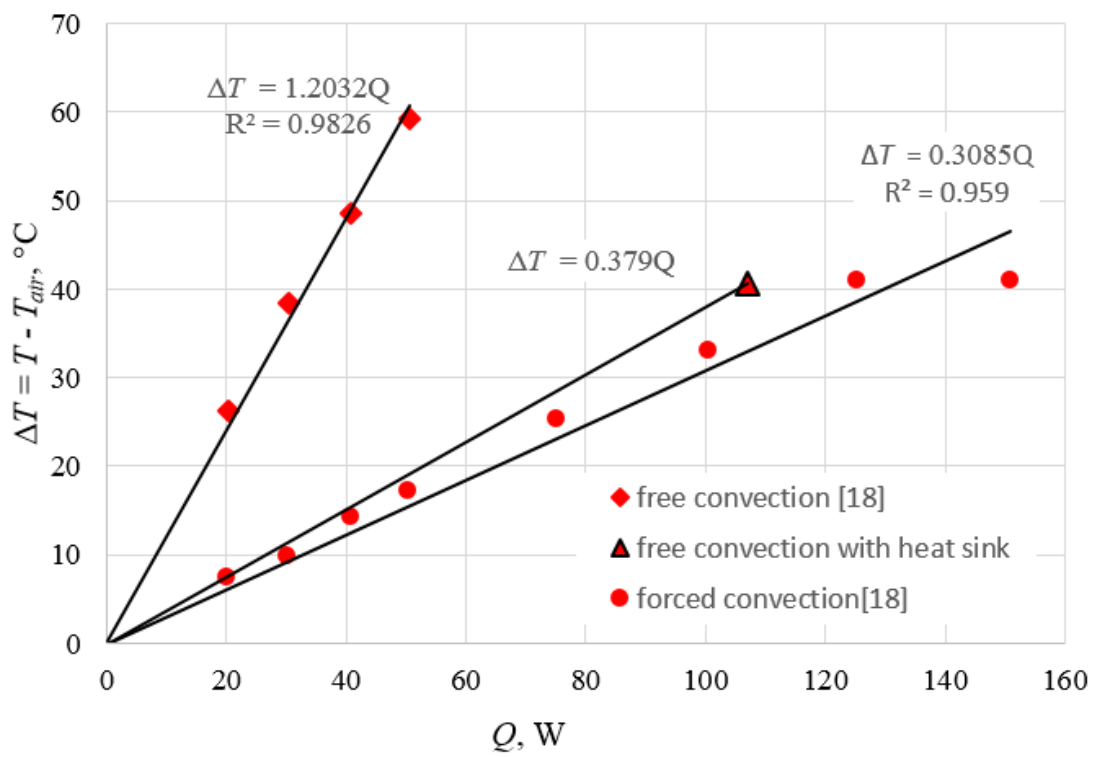

Fig. 4. Heat sink thermal resistance for free and forced convection

The heat sink attached by the manufacturer (fig. 1) provides much better heat transfer parameters than the heat sink used in [18]. However, the use of the active method of heat transfer enhancement allows more favorable operating conditions of the LED lamp. A significant disadvantage of the heat sink attached by the manufacturer is the considerable size of the entire device. As shown in Fig. 1, the heat sink has of considerable size, which reduces the possibility of mounting the lamp in places with limited space - the synthetic jet actuator investigated in [18] is smaller. Therefore, active forms of heat transfer should be sought.

\section{Conclusion}

The thermal power of LED lamps has been estimated and it is a reference point for further conclusions. It must be noted, that during the measurements the housing temperature was measured at a single point. In fact, the temperature distribution is uneven (approaching the LED junction, the temperature rises). The high value of the thermal given off by the housing of the LED lamp in comparison with the active power shows the low energy efficiency of the LED lamp. Time course of temperatures after switching on the LED lamp shows the difference between the air temperature and the temperature of the PCB near the SMD LEDs. After $40-50$ minutes, this difference is about $40-50^{\circ} \mathrm{C}$. However, the experimental studies were conducted at low air temperature. In practice, LED lamps are often used, for example, in production halls, where the air temperature is much higher, especially near the ceiling, i.e. where the lamps are placed. Then, taking into account the obtained temperature difference, the temperature value of the LED junction may significantly exceed the operating temperature recommended by the manufacturer, which, according to the research, will significantly worsen the parameters of the LEDs. Therefore, changes should be made to improve the parameters, e.g. by applying better cooling.

This work was supported by the National Center for Research and Development, Poland. Grant No.: LIDER/6/0024/L-10/18/NCBR/2019. 


\section{References}

1. X. Luo, R. Hu, S. Liu, and K. Wang, Prog. Energy Combust. Sci. 56, 1 (2016)

2. H. Ye, M. Mihailovic, C. K. Y. Wong, H. W. Van Zeijl, A. W. J. Gielen, G. Q. Zhang, and P. M. Sarro, Appl. Therm. Eng. 52, 353 (2013)

3. S. Chhajed, Y. Xi, T. Gessmann, J.-Q. Xi, J. M. Shah, J. K. Kim, and E. F. Schubert, Light. Diodes Res. Manuf. Appl. IX 5739, 16 (2005)

4. J. Hegedüs, G. Hantos, and A. Poppe, Energies 13, (2020)

5. A. Fan, R. Bonner, S. Sharratt, and Y. S. Ju, Annu. IEEE Semicond. Therm. Meas. Manag. Symp. 319 (2012)

6. S. Khandekar, G. Sahu, K. Muralidhar, E. Y. Gatapova, O. A. Kabov, R. Hu, X. Luo, and L. Zhao, Appl. Therm. Eng. 182, 115640 (2020)

7. H. Jang, J. H. Lee, C. Byon, and B. J. Lee, Int. J. Heat Mass Transf. 124, 36 (2018)

8. Y. Takata, S. Hidaka, J. M. Cao, T. Nakamura, H. Yamamoto, M. Masuda, and T. Ito, Energy 30, 209 (2005)

9. S. Mahmoud, A. Tang, C. Toh, R. AL-Dadah, and S. L. Soo, Appl. Energy 112, 1349 (2013)

10. H. Baumgartner, A. Vaskuri, P. Kärhä, and E. Ikonen, Appl. Therm. Eng. 71, 317 (2014)

11. C. S. Sharma, M. K. Tiwari, S. Zimmermann, T. Brunschwiler, G. Schlottig, B. Michel, and D. Poulikakos, Appl. Energy 138, 414 (2015)

12. M. A. Chan, C. R. Yap, and K. C. Ng, Int. J. Heat Mass Transf. 52, 3456 (2009)

13. K. P. Drummond, D. Back, M. D. Sinanis, D. B. Janes, D. Peroulis, J. A. Weibel, and S. V. Garimella, Int. J. Heat Mass Transf. 117, 319 (2018)

14. C. T. Nguyen, G. Roy, C. Gauthier, and N. Galanis, Appl. Therm. Eng. 27, 1501 (2007)

15. L. Lin and R. Ponnappan, Int. J. Heat Mass Transf. 46, 3737 (2003)

16. T. Yeom, L. Huang, M. Zhang, T. Simon, and T. Cui, Int. J. Heat Mass Transf. 143, 118484 (2019)

17. S. F. Liu, R. T. Huang, W. J. Sheu, and C. C. Wang, Int. J. Heat Mass Transf. 52, 2565 (2009)

18. P. Gil, E3S Web Conf. 100, 3 (2019)

19. P. Gil and J. Wilk, Int. J. Therm. Sci. 147, (2020)

20. P. Gil and E. Smyk, Sensors Actuators, A Phys. 295, 405 (2019)

21. E. Smyk, P. Gil, R. Gałek, and Ł. Przeszłowski, Actuators 9, 1 (2020) 